A Serial Mediation Model of the Relationship between Alexithymia and BMI: The Role of Negative Affect, Negative Urgency and Emotional Eating Aimee E Pink ${ }^{\mathrm{a}}$, Michelle Lee ${ }^{\mathrm{a}}$, Menna Price $^{\mathrm{a}} \&$ Claire Williams, ${ }^{\mathrm{a}, *}$ ${ }^{a}$ Department of Psychology College of Human and Health Sciences Swansea University Singleton Park Swansea, SA2 8PP UK.

A.E.Pink@swansea.ac.uk; M.D.Lee@swansea.ac.uk; M.J.Price@swansea.ac.uk; Claire.Williams@swansea.ac.uk

Declarations of interest: None. This research did not receive any specific grant from funding agencies in the public, commercial, or not-for-profit sectors. 
Abstract

27

Difficulty identifying and describing emotions (alexithymia) has been related to impulsiveness and negative affect, emotional eating and obesity. However, previous research

findings concerning the relationship between alexithymia and obesity have been mixed and inconsistent, raising the possibility that the relationship is indirect and mediated by multiple unknown factors. The aim of the study was to comprehensively explore the potential pathways between alexithymia and obesity via a novel theoretical model, and for the first time, incorporate negative affect, impulsiveness and emotional eating as potential mediating factors. Two questionnaire-based studies were conducted; the first as an exploratory analysis within a student sample $(N=125)$, and the second as a self-replication within a more representative general population sample $(N=342)$. Study One revealed that difficulty identifying feelings predicted Body Mass Index (BMI) both directly $(B=.1694, C I=.0194-$ $.3194)$ and indirectly via impulsiveness and emotional eating $(B=.0074, C I=.0001-.0315)$. In contrast, Study Two revealed that alexithymia predicted BMI indirectly via negative affect (when depression was included in the model; $B=.0335, C I=.0019-.0660$ ) or impulsiveness (when anxiety was included in the model; $B=.0021, C I=.0001-.0066$ ). Our findings provide partial support for the hypothesised model and offer original insight into the relationship between alexithymia and obesity. Additionally, our findings highlight important methodological considerations for future research and suggest that ways to address an individual's ability to identify, describe and regulate emotions should be considered when designing interventions to assist weight loss and management.

Keywords: Alexithymia, Emotional Dysregulation, Negative Urgency, Affect, Emotional Eating, BMI 

negative emotions (Pinaquy, Chabrol, Simon, Louvet, \& Barbe, 2003; Macht \& Simons, 2000). Generally, individuals who self-report as emotional eaters using validated measures, have been found to be at risk of weight gain from overconsumption, and in turn, increased body mass index (BMI) and obesity in the long term (Finch \& Tomiyama, 2015; Keonders \& van Strien, 2011; Kaplan \& Kaplan, 1957; Sung, Lee \& Song, 2009; van Strien, Herman \& Verheijdan, 2012). However, at an individual level, the relationship may vary depending on how individuals manage their eating behaviours and weight (e.g. Geliebter \& Aversa, 2003). Numerous theories of emotional eating have been proposed, including escaping from negative affect (Heatherton \& Baumeister, 1991), alleviating negative emotions (Lehman \& Rodin, 1989), and as a distraction mechanism (Polivy \& Herman, 1999). These theories all involve a maladaptive response to negative affect, and therefore, difficulties processing emotions could influence the behaviour.

One way in which the mechanisms underpinning emotional eating can be explored is through the examination of emotional dysregulation. Emotional dysregulation involves the combination of emotional vulnerability and the inability to modulate emotional responses three elements: difficulty identifying and describing emotions; the regulation of emotions, and the consequent behaviours (such as emotional eating; Spence \& Courbasson, 2012). Despite receiving little attention in the research literature, initial evidence suggests that emotional dysregulation may play an important role in eating disorders. Holliday, Uher, Landau, Collier and Treasure (2006) found higher levels of emotional dysregulation in women with anorexia nervosa compared to age-matched healthy controls, with similar findings reported in a sample of individuals with co-morbid eating and substance misuse 
emotional dysregulation may also play a role more broadly in eating behaviours, and specifically, emotional eating. However, this has yet to be comprehensively examined or explored in non-clinical samples.

Of particular relevance to emotional dysregulation is alexithymia, a personality trait characterised by difficulty identifying feelings and distinguishing them from the somatic sensations accompanying emotion (DIF), difficulty describing feelings to other people (DDF), constricted imaginal processes, and a stimulus-bound, externally oriented thinking style (EOT; Sifneos, 1973; Taylor, Bagby, \& Parker, 1997). Alexithymia has been shown to be highly prevalent in a number of medical and clinical conditions, particularly those characterised by poor affect regulation and the use of maladaptive coping strategies (e.g. Kang, Namkoong, Yoo, Jhung, \& Kim, 2012; Shishido, Gaher, \& Simons, 2013). For this reason, alexithymia may also be associated with emotional eating.

Consistent with this, Spence and Courbasson (2012) found that difficulty identifying and describing emotion was related to poor coping expectancies, which in turn, predicted emotional eating. In a sample of individuals with obesity, Larsen and colleagues (2006) also found higher levels of emotional eating to be associated with higher levels of alexithymia. Greater levels of alexithymia have also been found in individuals with obesity and binge eating disorder compared to those without, and alexithymia scores were also found to predict $17 \%$ of the variance in emotional eating scores, with DIF being the strongest significant unique predictor (Pinaquy et al., 2003). The relationship has also been tested experimentally, with higher levels of alexithymia associated with greater consumption of food following a distress induction task in healthy females (van Strien \& Ouwens, 2007).

In contrast, Noli and colleagues (2010) previously found a similar frequency of emotional eating in samples of severely obese individuals with and without alexithymia. However, they also found elevated levels of cognitive restraint, disinhibition and hunger in 
those with alexithymia, suggesting the presence of some degree of disordered eating behaviour in that group. It is possible that the use of an invalidated measure of emotional eating and the highly specific and complex sample studied, accounts, at least in part, for these mixed findings. Even so, Źak-Golab et al. (2013) also found no significant alexithymia differences between participants with obesity, with or without binge eating disorder, and a recent meta-analysis found increased DIF and EOT scores, but not DDF, in people with obesity compared with control groups (Fernandes et al., 2018). Therefore, whilst there appears to be some empirical evidence for a role of alexithymia in emotional eating and obesity, findings are mixed and inconsistent across similar samples, potentially raising the possibility that the relationships are mediated by multiple unknown factors which have yet to be considered.

In light of this, our aim is to comprehensively explore the mechanisms underpinning the relationship between alexithymia and emotional eating, expanding this further to explain variance in BMI. Based on available evidence, we propose a novel theoretical model that incorporates both negative affect and impulsivity as theoretically driven, mediating factors. Firstly, emotional eating is grounded in the idea that the consumption of food follows an emotional experience (van Strien, Frijters, \& Bergers, 1986). Therefore, it seems logical that an individual's level of general negative affect may play a role. Alexithymia has also been associated with increased levels of depression and anxiety (Berardis et al., 2008), and depression has been shown to be related to emotional eating in both clinical and general populations (e.g. Larsen, van Strien, Eisinga, \& Engels, 2006, Konttinen, Männistö, SarlioLähteenkorva, Silventoinen, \& Haukkala, 2010). In addition, a study conducted in two European countries found that emotional eating mediated the relationship between depression and BMI (van Strien, Konttinen, Homberg, Engels, \& Winkens, 2016). It could therefore be 
theorised that individuals with poor emotional regulation may be at increased risk of negative affect, which in turn, may exacerbate their emotional eating.

Secondly, impulsive tendencies have been associated with emotional eating (Jansinka et al., 2012), and specifically, negative urgency which is defined as acting rashly in response to negative affect (as measured by the UPPS-P; Cyders, Smith, Spillane, Fischer, Annus, \& Peterson, 2007; Pike, 2013). In a sample of female twins, Racine et al. (2013) found a moderate significant correlation between negative urgency and emotional eating, even after controlling for the effects of negative affect. Etiological and twin model results also revealed that genetic and, to a lesser extent, non-shared environmental factors accounted for this relationship. Further, the genetic factors influencing negative urgency and dysregulated eating were highly correlated; leading to the conclusion that negative urgency likely increases the risk of developing binge and emotional eating through primarily genetic mechanisms. Such findings suggest that individuals may be most at risk of engaging in emotional eating if they experience elevated levels of affect and make rash decisions in response. In addition, Pike (2013) reported a significant positive relationship between alexithymia and negative urgency in a sample of undergraduate students, finding that both factors positively correlated with emotional eating. However, negative urgency did not significantly predict emotional eating or moderate its relationship with alexithymia, suggesting that the relationship is complex and that other factors likely play a role.

To date, only one study has attempted to explore the relationship between depression and emotional eating including impulsivity and the DIF facet of alexithymia as possible indirect pathways (Ouwens, van Strien, \& van Leeuwe, 2009). Ouwens and colleagues (2009) found that DIF, impulsivity and depression were all significantly correlated with emotional eating, and that depression was directly and indirectly (through DIF and impulsivity) related to emotional eating. In contrast, in our model we seek to explore negative urgency as a more 
specific facet of impulsivity, to examine negative affect in terms of both depression and anxiety, and to explore the alexithymia construct as a whole, as well as its three core features. In summary, we sought to conduct the first comprehensive examination of the potential mediating pathways between alexithymia and BMI via a novel theoretical model. Potential mediators considered were negative affect, impulsiveness and emotional eating (see Figure 1). To address this in a robust manner, we conducted an exploratory analysis in a student sample (Study One) and a self-replication to test the applicability of the model in a more representative general population sample (Study Two). Our model (see Figure 1) theorises that alexithymia is indirectly predictive of BMI. We propose that individuals who have difficulty understanding their emotions (alexithymia) are vulnerable to experiencing increased levels of anxiety and depression (negative affect), which may cause them to act rashly in response (negative urgency) and engage in emotional eating, leading to a negative impact on BMI.

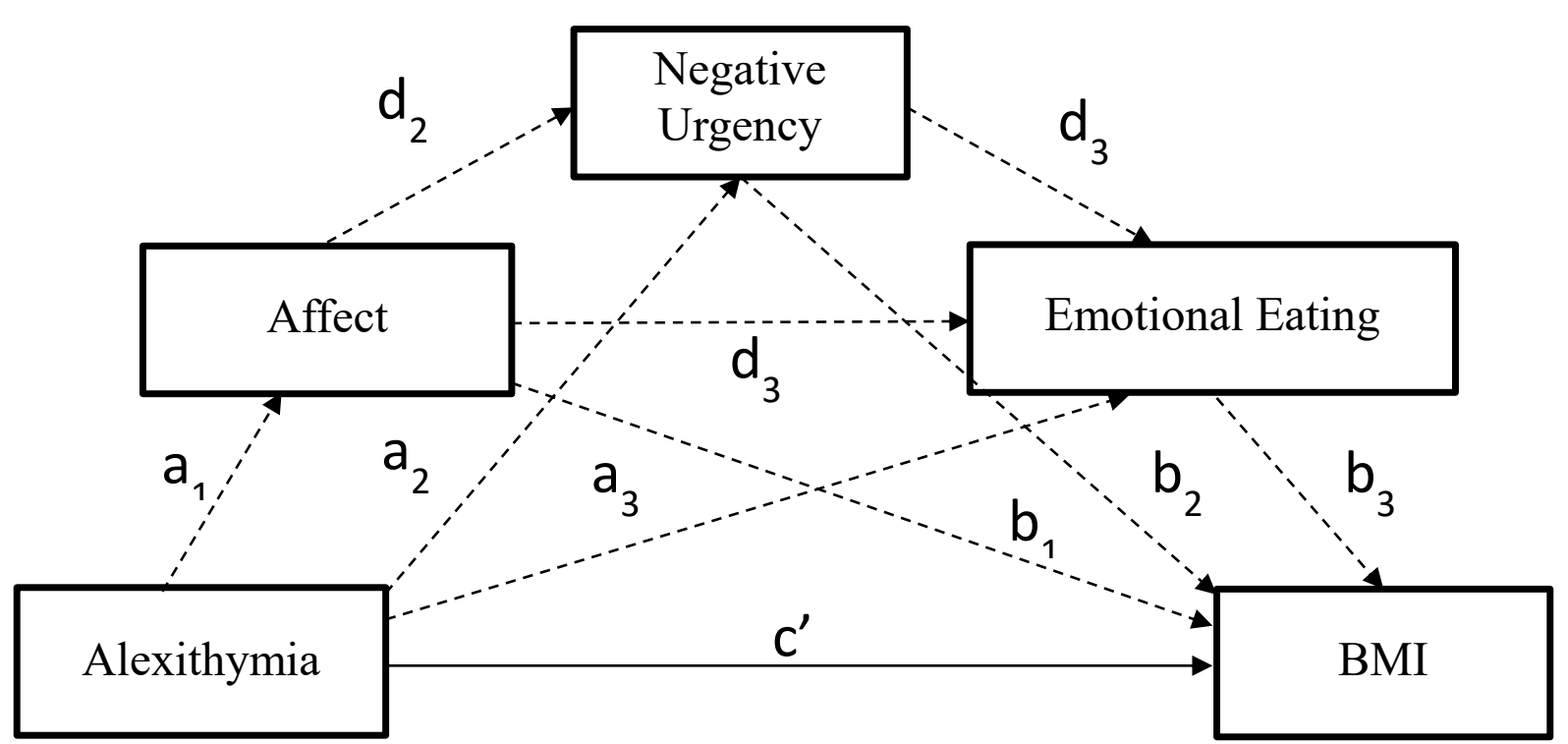

Figure 1. Theoretical model of emotional eating inclusive of alexithymia, affect and impulsivity. 


\section{Study One}

Here we explore the model in a student sample as part of an exploratory analysis.

\section{Method}

\section{Participants}

The sample consisted of students drawn from Swansea University who participated for course credit or volunteered in response to study adverts. Participants had to be between 18-65 years of age and report no history of eating, mood, addictive or substance use disorders, with eligibility determined as part of the consent process. 130 participants initially met these criteria. However, five participants were subsequently removed owing to a reported history of eating disorders (disclosed after completion) or missing height and weight data which meant BMI could not be calculated.

Of the remaining 125 participants, $107(85.60 \%)$ were female and the mean age of the sample was 20.85 years of age $(S D=3.06$, range $=18.27-36.87$ years $) .115$ $(92.00 \%)$ participants were single, with the remaining $10(8.00 \%)$ co-habiting. Participants had spent an average of 14.30 years in education $(S D=.92$, range $12-18$ years $)$ and $90.40 \%$ $(n=113)$ reported their ethnic background as White. Remaining participants reported their ethnic backgrounds as Asian $(n=3,2.4 \%)$, Black $(n=2,1.6 \%)$, Mixed $(n=6,4.8 \%)$ and other $(n=1,0.8 \%)$. Mean BMI was $23.99(\mathrm{SD}=4.34)$ with values ranging from 16.84 to $41.21 \mathrm{~kg} / \mathrm{m}^{2}$.

\section{Measures}



status, ethnicity and years spent in education.

Toronto Alexithymia Scale (TAS-20; Bagby, Parker, \& Taylor, 1994; Bagby, Taylor,

\& Parker, 1994): Regarded as the gold-standard method of assessing alexithymia, the TAS-20 consists of 20 items measuring three facets of alexithymia: difficulty identifying feelings (DIF; e.g. "I am often confused about what emotion I am feeling”), difficulty describing feelings (DDF; e.g. "It is difficult for me to find the right words for my feelings"), and externally-oriented thinking (EOT; e.g. "Being in touch with emotions is essential”). Participants indicate their responses by selecting the answer that best describes them on a 5point scale $(1=$ strongly disagree to $5=$ strongly agree). The three subscales (DIF, DDF, EOT) are combined to generate a total TAS-20 alexithymia score which can be used to determine the presence or absence of alexithymia. Scores $\geq 6$ indicate the presence of alexithymia, scores of 52-60 indicate 'possible' alexithymia and scores $\leq 51$ indicate the absence of alexithymia (Taylor, Bagby, \& Parker, 1992). The TAS-20 has good levels of validity (Bagby, Taylor, et al., 1994) and in line with previous research (Bagby, Parker, et al., 1994; Pinaquy et al., 2003), internal consistency was found to be high in the current study ( $\alpha$ $=.84)$. High levels of internal consistency were also found for the DIF $(\alpha=.83)$ and DDF $(\alpha$ $=.80$ ) subscales. Consistent with previously reported levels (Larsen et al., 2006; Pike, 2013; Pinaquy et al., 2003), internal consistency of the EOT subscale was much lower $(\alpha=.55)$.

UPPS-P Impulsivity Behaviour Scale (UPPS-P; Cyders et al., 2007; Whiteside \& Lynam, 2001): The (negative) urgency, (lack of) premeditation, (lack of) perseverance, sensation seeking and positive urgency measure of impulsivity. However, the current study only reports data from the negative urgency subscale (NU, 12 items; e.g. "When I feel bad, I will often do things I later regret in order to make myself feel better now”). Participants respond on a four-point Likert type scale ( $1=$ agree strong to $4=$ disagree strongly), with 
higher scores indicating greater impulsive tendencies. The Cronbach's alpha for the NU subscale was $\alpha=.87$, and whilst other measures of impulsivity are available, the UPPS-P was chosen as it has a specific measure of NU.

Beck Depression Inventory - II (BDI; Beck, Ward, Mendelson, Mock, \& Erruagh, 1961; Beck, Steer, \& Carbin, 1988; Beck, Steer, \& Brown, 1996): The BDI is the most commonly used measure of depression, consisting of 21 items that assess the severity of depressive symptoms experienced in the last two weeks. Respondents choose an answer that best describes them on a four-point Likert-type scale $(0=$ not at all, no change to $3=$ increase/decrease in symptom) with statements referring to "sadness," "pessimism," and "past failure," as examples. The maximum score on the BDI is 63, with scores between 0-13 indicating the presence of minimal depression, 14-19 mild depression, 20-28 moderate depression, and 29-63 severe depression (Wood, Williams, \& Lewis, 2010). Internal consistency was found to be high $(\alpha=.90)$ within the current study.

Beck Anxiety Inventory (BAI; Beck, Epstein, Brown, \& Steer, 1988): Consisting of 21-items, the BAI asks participants to rate how much they have been bothered by emotional, physiological, and cognitive symptoms of anxiety in the last week. Participants respond by selecting the answer that best describes them on a four-point Likert-type scale $(0=$ not at all to $3=$ severely). Total scores range between $0-63$, with scores between $0-9$ indicating the absence of anxiety, 10-18 mild to moderate anxiety; 19-29 moderate to severe anxiety, and 30-63 severe anxiety (Beck, \& Steer, 1990). The BAI was specifically chosen over other measures as it has excellent psychometric properties and is widely used in both research and clinical practice (Beck, Epstein et al., 1988). In line with previous research (Beck, Epstein, et al., 1998), internal consistency was high $(\alpha=.91)$. 
242 Sjöström, \& Sullivan, 2000): The TFEQ is a revised shortened revision of the TFEQ

243 (Stunkard \& Messick, 1985). Previous dichotomous responses were replaced with Likert type scales as the former were found to be restricting. Participants respond by selecting the answer that best describes them on a 4 -point Likert type scale $(1=$ definitely true to $4=$ definitely false). The TFEQ-R18 consists of three subscales: cognitive restraint (e.g. "I do not eat some foods because they make me fat"); uncontrolled eating (e.g. "I get so hungry that my stomach often seems like a bottomless pit"), and emotional eating (e.g. "When I feel anxious, I find myself eating"). 13 items are reversed scored and higher total scores are indicative of greater levels of cognitive restraint, uncontrollable eating and emotional eating. Although developed in an obese population, the TFEQ-R18 has been found to valid within general population samples and to have satisfactory levels of internal consistency (de Lauzon, et al., 2004). In the current study, the following Cronbach's alphas were found: cognitive restraint $(\alpha=.86)$, uncontrollable eating $(\alpha=.88)$, and emotional eating $(\alpha=.85)$. The TFEQ was selected to allow the inclusion of cognitive restraint and uncontrolled eating scores as control variables in the serial multivariate mediation analyses.

Emotional Eating Scale (EES; Arnow, Kernady, \& Agras, 1995): The EES is a 25 self-report measure to explore the urge to eat in response to a number of emotions (e.g. "Lonely" and "Angry"). Participants respond by selecting the answer that best describes them on a fivepoint scale ( $1=$ no desire to eat, to $5=$ an overwhelming urge to eat). In addition to an EES total score, subscale scores can be calculated for anger/frustration, anxiety, and depression. The EES demonstrated adequate to excellent levels of internal consistency (range $\alpha=.75$ .93), and although initially created within a clinical population, it has been used extensively in general population samples (e.g. Price, Higgs, \& Lee, 2015; Moon \& Berenbaum, 2009). The EES was favoured over alternative measures of emotional eating (i.e., Dutch Eating 
Behaviour Questionnaire; van Strien et al., 1986) as it captures eating in response to a wide

267 range of negative emotions.

Body Mass Index (BMI); obtained by taking a measurement of height using a stadiometer in metres and three readings of weight to gain an average using WW digital scales in kilograms. The BMI value was calculated using the following equation in excel (weight $(\mathrm{kg}) /\left(\right.$ height $\left.(\mathrm{m})^{2}\right)$ ).

\section{Procedure}

Ethical approval was obtained from the Department of Psychology Research Ethics Committee, College of Human and Health Sciences, Swansea University. Participants were presented with a detailed information sheet before providing written informed consent to partake. All participants also completed a delayed discounting task which was part of a larger study, although data from this task is not reported here. Participants were then instructed to work through the self-reported questionnaires presented to them via Survey Monkey (Palo Alto, California, USA). Upon completion, weight and height measurements were taken so BMI could be calculated. The study lasted for approximately 30 minutes and participants were thanked for their time upon completion.

\section{Data Analysis}

All statistical analyses were carried out using IBM SPSS Statistics 22.0 and PROCESS 2.16.3 (Hayes, 2013). Preliminary analysis examined the presence of outliers and the assumptions of normality were met. In terms of statistical analyses employed, Pearson correlations were used to investigate the associations between measures and PROCESS was used to test our theorised models. PROCESS was used as significant associations between 
variables are not needed and bootstrapping reduces type 1 error. Finally, all variables (including TAS-20, BDI and BAI) were treated as continuous variables.

Serial multivariate mediation analyses.

291

292

293

A series of exploratory serial multiple mediation models (Hayes, 2013) were conducted. 16 models were analysis for direct and specific indirect effects (see Table 1). A direct effect $\left(c^{\prime}\right)$ is the relationship between $X$ and $Y$ controlling for all mediators, and a specific indirect effect (e.g. $a_{1} b_{1}, a_{1} d_{21} b_{2}$ ) is the relationship between $X$ and $Y$ via a particular mediator or mediators. Using Model 1 (see Table 1) and Figure 1, the direct and indirect effects analysed can be demonstrated. Total alexithymia scores were entered as the predictor variable $(X)$ and BMI scores as the outcome variable $(Y)$. Multiple mediators were then entered in the following order; negative affect, impulsivity (negative urgency) and emotional eating, e.g. Model 1; BDI $\rightarrow$ NU $\rightarrow$ TFEQ-EE. This created and tested the following indirect pathways; via BDI alone $\left(a_{1} b_{1}\right)$, via BDI and NU $\left(a_{1} d_{2 l} b_{2}\right)$, via NU only $\left(a_{2} b_{2}\right)$, via NU and TFEQ-EE $\left(a_{2} d_{32} b_{3}\right)$, via BDI and TFEQ-EE $\left(a_{1} d_{31} b_{3}\right)$, via TFEQ-EE only $\left(a_{3} b_{3}\right)$ and finally via BDI, NU and TFEQ-EE $\left(a_{1} d_{21} d_{32} b_{3}\right)$. The remaining models followed this structure substituting the TAS-20 subscales for $X$, BAI for affect at $M_{1}$ and emotional eating as measured by EES at M3. Mediating variables are also controlled for in indirect effects. 95\% bias-corrected confidence intervals based on 10,000 bootstrap samples were calculated. Finally, as age, gender, uncontrolled eating and cognitive restraint have all been correlated with emotional eating and BMI (e.g. Koenders \& van Strien, 2011; Larsen et al., 2006; Lluch, Herbeth, Méjean \& Siest, 2000); these variables were controlled for in all models (e.g. younger participants may have a lower BMI compared to older subjects simply because not enough time has passed since the onset of emotional eating behaviours, rather than reflecting the influence of predictor variables). 


\begin{tabular}{|c|c|}
\hline Model & Pathway tested \\
\hline 1 & $\mathrm{TAS}-20 \rightarrow \mathrm{BDI} \rightarrow \mathrm{NU} \rightarrow \mathrm{TFEQ}-\mathrm{EE} \rightarrow \mathrm{BMI}$ \\
\hline 2 & $\mathrm{DIF} \rightarrow \mathrm{BDI} \rightarrow \mathrm{NU} \rightarrow \mathrm{TFEQ}-\mathrm{EE} \rightarrow \mathrm{BMI}$ \\
\hline 3 & $\mathrm{DDF} \rightarrow \mathrm{BDI} \rightarrow \mathrm{NU} \rightarrow \mathrm{TFEQ}-\mathrm{EE} \rightarrow \mathrm{BMI}$ \\
\hline 4 & $\mathrm{EOT} \rightarrow \mathrm{BDI} \rightarrow \mathrm{NU} \rightarrow \mathrm{TFEQ}-\mathrm{EE} \rightarrow \mathrm{BMI}$ \\
\hline 5 & $\mathrm{TAS}-20 \rightarrow \mathrm{BDI} \rightarrow \mathrm{NU} \rightarrow \mathrm{EES} \rightarrow \mathrm{BMI}$ \\
\hline 6 & $\mathrm{DIF} \rightarrow \mathrm{BDI} \rightarrow \mathrm{NU} \rightarrow \mathrm{EES} \rightarrow \mathrm{BMI}$ \\
\hline 7 & $\mathrm{DDF} \rightarrow \mathrm{BDI} \rightarrow \mathrm{NU} \rightarrow \mathrm{EES} \rightarrow \mathrm{BMI}$ \\
\hline 8 & $\mathrm{EOT} \rightarrow \mathrm{BDI} \rightarrow \mathrm{NU} \rightarrow \mathrm{EES} \rightarrow \mathrm{BMI}$ \\
\hline 9 & $\mathrm{TAS}-20 \rightarrow \mathrm{BAI} \rightarrow \mathrm{NU} \rightarrow \mathrm{TFEQ}-\mathrm{EE} \rightarrow \mathrm{BMI}$ \\
\hline 10 & $\mathrm{DIF} \rightarrow \mathrm{BAI} \rightarrow \mathrm{NU} \rightarrow \mathrm{TFEQ}-\mathrm{EE} \rightarrow \mathrm{BMI}$ \\
\hline 11 & $\mathrm{DDF} \rightarrow \mathrm{BAI} \rightarrow \mathrm{NU} \rightarrow \mathrm{TFEQ}-\mathrm{EE} \rightarrow \mathrm{BMI}$ \\
\hline 12 & $\mathrm{EOT} \rightarrow \mathrm{BAI} \rightarrow \mathrm{NU} \rightarrow \mathrm{TFEQ}-\mathrm{EE} \rightarrow \mathrm{BMI}$ \\
\hline 13 & $\mathrm{TAS}-20 \rightarrow \mathrm{BAI} \rightarrow \mathrm{NU} \rightarrow \mathrm{EES} \rightarrow \mathrm{BMI}$ \\
\hline 14 & $\mathrm{DIF} \rightarrow \mathrm{BAI} \rightarrow \mathrm{NU} \rightarrow \mathrm{EES} \rightarrow \mathrm{BMI}$ \\
\hline 15 & $\mathrm{DDF} \rightarrow \mathrm{BAI} \rightarrow \mathrm{NU} \rightarrow \mathrm{EES} \rightarrow \mathrm{BMI}$ \\
\hline 16 & $\mathrm{EOT} \rightarrow \mathrm{BAI} \rightarrow \mathrm{NU} \rightarrow \mathrm{EES} \rightarrow \mathrm{BMI}$ \\
\hline
\end{tabular}

$\mathrm{BMI}=$ body mass index, DIF = difficulty identifying feelings, DDF = difficulty describing feelings, EOT = externally oriented thinking, TAS-20 = total alexithymia scores, $\mathrm{NU}=$ negative urgency, $\mathrm{BDI}=$ Beck depression inventory, $\mathrm{BAI}=$ Beck anxiety inventory, $\mathrm{EES}=$ total emotional eating scale, TFEQ-EE = emotional eating subscale from the TFEQ.

\section{Results}

\section{Descriptives}

Mean total scores were as follows: TAS-20: $M=45.51, S D=11.33$; NU: $M=28.26$;

$S D=7.01 ;$ EES: $M=48.60, S D=16.41$;FEQ: $M=42.58, S D=29.15 ;$ BDI: $M=10.14, S D$

$322=8.08$, and BAI: $M=10.10 ; S D=9.49$ ). In addition, levels of alexithymia were consistent

with previously reported (Bagby, Parker et al., 1994; Salminen, Saarijärvi, Äärelä, Toikka, \& 
alexithymia: $n=23,18.4 \%$; no alexithymia: $n=89,71.2 \%$ ), and the majority of the sample reported minimal levels of depression (minimal/no depression: $n=91,72.8 \%$; mild: $n=18$, 14.4\%; moderate: $n=10,8.0 \%$; severe: $n=6,4.8 \%$ ) and anxiety (minimal/no anxiety: $n=$ 78, 62.4\%; mild: $n=26,20.8 \%$; moderate: $n=14,11.2 \%$; severe: $n=7,5.6 \%$ ).

\section{Correlation Analysis}

Pearson's correlations were conducted to initially explore the relationships between alexithymia, negative urgency, negative affect, emotional eating and BMI. Significant positive correlations were found between EES total scores and TAS-20 total scores $(r=.176$, $p=.049)$ and DIF subscale scores $(r=.203, p=.024$; see Table 2$)$. The negative urgency facet was also significantly positively correlated with EES total scores $(r=.385, p \leq .001)$, and similar results were found for the emotional eating subscale of the TFEQ $(r=.380, p \leq$ .001). Significant positive correlations were also found between BDI, BAI and emotional eating scores as measured by the EES and TFEQ-EE (see Table 2). There was no significant correlation between BMI and any of the other variables. When applying Bonferroni's correction $(p<.0011)$ several relationships between emotional eating, alexithymia, negative urgency and negative affect remained significant (see Table 2).

Table 2. Pearson's correlations of all study variables for the Study One.

\begin{tabular}{|c|c|c|c|c|c|c|c|c|}
\hline & 1. & 2. & 3. & 4. & 5. & 6. & 7. & 8. \\
\hline 1. DIF & - & & & & & & & \\
\hline 2. DDF & $.638 * * *$ & - & & & & & & \\
\hline 3. EOT & $.270 * *$ & $.331 * * *$ & - & & & & & \\
\hline $\begin{array}{l}\text { 4. TAS } \\
\text { Total }\end{array}$ & $.853 * * *$ & $.849 * * *$ & $\begin{array}{l}.631 \\
* * *\end{array}$ & - & & & & \\
\hline
\end{tabular}




\begin{tabular}{|c|c|c|c|c|c|c|c|c|c|}
\hline 5. NU & $.422 * * *$ & $234 * *$ & .102 & $\begin{array}{c}.341 * * \\
*\end{array}$ & - & & & & \\
\hline $\begin{array}{l}\text { 6. BDI } \\
\text { Total }\end{array}$ & $.529 * * *$ & $.397 * * *$ & .037 & $\begin{array}{c}.438 * * \\
*\end{array}$ & $\begin{array}{c}.379 * * \\
*\end{array}$ & - & & & \\
\hline $\begin{array}{l}\text { 7. BAI } \\
\text { Total }\end{array}$ & $.437 * * *$ & $.308 * * *$ & .116 & $\begin{array}{c}.384 * * \\
*\end{array}$ & $\begin{array}{c}.304 * * \\
*\end{array}$ & $\begin{array}{c}.639 * * \\
*\end{array}$ & - & & \\
\hline $\begin{array}{l}\text { 8. EES } \\
\text { Total }\end{array}$ & $.203 *$ & .085 & .115 & $.176^{*}$ & $\begin{array}{c}.385^{* *} \\
*\end{array}$ & $.265 * *$ & $.271 * *$ & - & \\
\hline $\begin{array}{l}\text { 9. TFEQ } \\
\text {-EE }\end{array}$ & .074 & .018 & .090 & .076 & $\begin{array}{c}.328 * * \\
*\end{array}$ & .069 & -.037 & $.588 * * *$ & - \\
\hline 10. BMI & .151 & .078 & $\begin{array}{c}- \\
.067\end{array}$ & .083 & .077 & .065 & .144 & .150 & .138 \\
\hline
\end{tabular}

343 DIF = difficulty identifying feelings, DDF = difficulty describing feelings, EOT = externally oriented thinking, TAS-20 $=$ total alexithymia scores, $\mathrm{NU}=$ negative urgency, $\mathrm{BDI}$ $=$ Beck depression inventory, $\mathrm{BAI}=$ Beck anxiety inventory, EES = total emotional eating scale, TFEQ-EE = emotional eating subscale from the TFEQ, BMI = body mass index.

\section{Serial Mediation Models}

Depression. The PROCESS macro was used to analyse the models presented in Table

1. Model 1 revealed no significant direct effect between alexithymia and BMI, but a significant specific indirect effect approached significance, via BDI, NU, and TFEQ-EE $\left(a_{1} d_{21} d_{32} b_{3}\right), B=.0009, C I=.0000-.0059$. Hayes (2013) states that confidence intervals must not contain 0 for an effect to be significant. Running the model with emotional eating, as measured by EES at $M_{3}$, revealed a similar pattern of results (Model 5). Model 2, DIF entered as $X$, showed a significant direct effect $\left(c^{\prime}\right), B=.1694, C I=.0194-.3194$ and one significant specific indirect effect via NU and TFEQ-EE $\left(a_{2} d_{31} b_{3}\right), B=.0074, C I=.0001-.0315 . \mathrm{A}$ second effect, via BDI, NU and TFEQ-EE $\left(a_{1} d_{21} d_{32} b_{3}\right), B=.0021, C I=.0000-.0129$, approached significance. When entering EES at $M_{3}$, Model 6 revealed a significant direct effect $\left(c^{\prime}\right), B=.1608, C I=.0107-.3109$ and a specific indirect effect via BDI, NU and EES $\left(a_{1} d_{21} d_{32} b_{3}\right), B=.0016, C I=.0000-.0103$ approached significance. There were no significant direct or indirect effects for models 3, 4, 7 and 8 . 
Anxiety. Analysis of model 9, entering BAI at $M_{l}$, found no significant direct effect between TAS-20 total scores and BMI. However, a significant specific indirect effect was found, via NU and TFEQ-EE $\left(a_{2} d_{31} b_{3}\right), B=.0034, C I=.0002-.013$. A second indirect effect approached significance, via BAI, NU and TFEQ-EE $\left(a_{1} d_{21} d_{32} b_{3}\right), B=.0008, C I=.0000-$ .0051. Model 10, with DIF entered as $X$, revealed one significant indirect effect via NU and TFEQ-EE $\left(a_{2} d_{32} b_{3}\right), B=.0102, C I=.0009-.0353$. Model 11 , including DDF as $X$, had no significant direct effect but three significant specific indirect effects. The first via BAI $\left(a_{1} b_{1}\right)$, $B=.0427, C I=.0014-.1162$, the second via BAI, NU, and TFEQ-EE $\left(a_{1} d_{21} d_{32} b_{3}\right), B=.0019$, $C I=.0001-.0119$, and the third via NU and TFEQ-EE $\left(a_{2} d_{31} b_{3}\right), B=.0052, C I=.0001-.0243$. Contrast analysis revealed the effects via BAI only was significantly stronger than via BAI, NU and TFEQ-EE $(\mathrm{B}=.0408, C I=.0003-.1118)$, with no difference between the latter and the effect via NU and TFEQ-EE. Models 12-16 did not reveal any significant direct or indirect effects between total and subscale scores of the TAS-20 $(X)$ and BMI $(Y)$.

\section{Discussion}

In our exploratory analysis of a student sample we found a significant direct effect of the difficulty identifying feelings subscale on BMI. Furthermore, a specific indirect effect suggests that difficulty identifying feelings can lead to an increased tendency to react rashly to negative affect (negative urgency), exacerbating emotional eating which could lead to an increased BMI.

When anxiety scores were included in the model, an indirect effect via negative urgency and emotional eating was found for total alexithymia scores and the difficulty identifying subscale, but only for emotional eating as measured by the TFEQ. The role of difficulty describing feelings appears to be stronger when anxiety is included in the model as 
three indirect effects were found to be significant. These effects were through the following pathways: via increased anxiety scores only; via increased negative urgency and emotional eating, and via increased anxiety, negative urgency and emotional eating. Contrast analysis revealed that the indirect pathway via increased anxiety scores was the strongest.

\section{Study Two}

Study Two sought to re-examine the relationships between alexithymia, negative affect, negative urgency, emotional eating and BMI, and to test the applicability of the significant models in Study One in a more representative general population sample. On the basis of our previous findings, significant positive correlations were predicted between alexithymia, negative affect, emotional eating and negative urgency. In addition, similar indirect and direct effects between DIF and BMI were expected to emerge when depression was included in the models. In contrast, we expected DDF to have a significant indirect effect on BMI via multiple pathways (e.g. via anxiety alone; via negative urgency and emotional eating) when anxiety was included in the models.

\section{Participants}

Invitations to take part in an online study were advertised on social media and via Swansea University's online platforms. As with Study One, participants had to be between 18-65 years of age and report no history of eating, mood, addictive, or substance misuse disorders, with eligibility determined as part of the consent process. 651 participants initially accessed the survey, although data for 309 participants was subsequently removed because of missing, inaccurate or incomplete data (e.g. non-completers), or because of failure to provide 
full consent and/or meet eligibility criteria. Of the remaining 342 participants, mean age was 32.36 years old $(S D=11.38$, range $=18.20-64.13) .279(81.6 \%)$ were female and the majority $(n=315,92.1 \%)$ reported their ethnicity as white, with other ethnicities reported being Asian $(n=7,2 \%)$, Black $(n=5, .9 \%)$, Chinese $(n=2, .6 \%)$, Mixed $(n=8,2.3 \%)$ and other, or preferred not to say $(n=7,2 \%)$. BMI ranged from 16.33-48.10 with an average of $25.94(S D=5.51)$.

\section{Measures}

The same measures as Study One were employed. However, as this was an online study, BMI was calculated from self-reported height and weight.

\section{Procedure}

Ethical approval was obtained from the Department of Psychology Ethics Committee, College of Human and Health Sciences, Swansea University. Participants completed the study online via Survey Monkey (Palo Alto, California, USA) in their own time. Participants were presented with an information page and upon providing full consent on screen, were asked to answer standard demographic questions followed by the TAS-20, BDI, BAI, UPPSP, EES and TFEQ. Participants were also asked for their height and weight and were provided with conversion details to report both in metric format. If participants wanted to withdraw at any time they could do so by closing the web browser. Upon completion, participants were presented with a debrief page and were thanked for their time.

\section{Data Analysis}

Statistical analysis was carried out using SPSS 22.0 and PROCESS 2.16.3 (Hayes, 2013). As all variables were normally distributed, Pearson's correlation analysis was performed. Serial mediation analysis was conducted and as per Study One, age, gender, 
uncontrolled eating and cognitive restraint scores were controlled for in all models. In addition, all variables were included in the models as continuous variables.

\section{Descriptives}

\section{Results}

Mean total scores were as follows: TAS-20: $M=46.84, S D=13.33$; NU: $M=9.42$; $S D=7.44$; EES: $M=50.88, S D=19.79$; TFEQ: $M=57.76, S D=22.31 ; \mathrm{BDI}: M=10.66, S D$ $=9.77$, and BAI: $M=10.66 ; S D=9.49)$. The majority of the sample reported an absence of clinically relevant alexithymia (Alexithymia: $n=61,17.8 \%$; possible alexithymia: $n=61$, 17.8\%; no alexithymia: $n=220,64.3 \%$ ), and minimal levels of depression (minimal/no depression: $n=213,62.3 \%$; mild: $n=49,14.3 \%$; moderate: $n=49,14.3 \%$; severe: $n=31$, 9.1\%) and anxiety (minimal/no anxiety: $n=200,58.5 \%$; mild: $n=75,21.9 \%$; moderate: $n=$ 48, 14.0\%; severe: $n=19,5.6 \%)$.

\section{Correlation Analysis}

Pearson's correlations showed that TAS-20 total, DIF and DDF scores were significantly positively correlated with emotional eating as measured by the EES and the TFEQ (see Table 3). Negative urgency was positively and significantly correlated with emotional eating; EES: $r=.350, p<.001$ and TFEQ: $r=.324, p<.001$. There was also a positive significant relationship between BDI scores and emotional eating as measured by both the EES and TFEQ (see Table 3). BAI scores were only significantly related to EES total scores. BMI scores were not significantly related to alexithymia but were weakly correlated with negative urgency $(r=.152, p=.005)$ and emotional eating (EES: $r=.212, p<$ 
.001 , TFEQ: $r=.2, p<.001)$. Several relationships remained significant after applying a

457 Bonferroni adjusted alpha of $p=.0011$ (See Table 3).

459 Table 3. Pearson's correlations of all study variables for the Study Two.

\begin{tabular}{|c|c|c|c|c|c|c|c|c|c|}
\hline & 1. & 2. & 3. & 4. & 5. & 6. & 7. & 8. & 9. \\
\hline 1. DIF & - & & & & & & & & \\
\hline 2. DDF & $\begin{array}{c}.706 * \\
* *\end{array}$ & - & & & & & & & \\
\hline 3. EOT & $\begin{array}{c}.275 * \\
* *\end{array}$ & $\begin{array}{c}.453 * * \\
*\end{array}$ & - & & & & & & \\
\hline $\begin{array}{l}\text { 4. TAS- } \\
20\end{array}$ & $\begin{array}{c}.858^{*} \\
* *\end{array}$ & $\begin{array}{c}.887^{* *} \\
*\end{array}$ & $\begin{array}{c}.670 * * \\
*\end{array}$ & - & & & & & \\
\hline $\begin{array}{l}\text { Total } \\
\text { 5. NU }\end{array}$ & $\begin{array}{c}.524^{*} \\
* *\end{array}$ & $\begin{array}{c}.396 * * \\
*\end{array}$ & $\begin{array}{c}.214 * * \\
*\end{array}$ & $\begin{array}{c}.485 * * \\
*\end{array}$ & - & & & & \\
\hline $\begin{array}{l}\text { 6. BDI } \\
\text { Total }\end{array}$ & $\begin{array}{c}.645^{*} \\
* *\end{array}$ & $\begin{array}{c}.516^{* * *} \\
*\end{array}$ & $\begin{array}{c}.213 * * \\
*\end{array}$ & $\begin{array}{c}.589 * * \\
*\end{array}$ & $\begin{array}{c}.544 * * \\
*\end{array}$ & - & & & \\
\hline $\begin{array}{l}\text { 7. BAI } \\
\text { Total }\end{array}$ & $\begin{array}{c}.553 * \\
* *\end{array}$ & $.396 * *$ & $.118^{*}$ & $\begin{array}{c}.464 * * \\
*\end{array}$ & $\begin{array}{c}.354 * * \\
*\end{array}$ & $\begin{array}{c}.699 * * \\
*\end{array}$ & - & & \\
\hline $\begin{array}{l}\text { 8. EES } \\
\text { Total }\end{array}$ & $\begin{array}{c}.265^{*} \\
* *\end{array}$ & $\begin{array}{c}.174 * * \\
*\end{array}$ & .058 & $.217 * *$ & $\begin{array}{c}.350 * * \\
*\end{array}$ & $\begin{array}{c}.317 * * \\
*\end{array}$ & $\begin{array}{c}.241 * * \\
*\end{array}$ & - & \\
\hline $\begin{array}{l}\text { 9. TFEQ } \\
\text {-EE }\end{array}$ & $\begin{array}{l}.180^{*} \\
* *\end{array}$ & $.218 *$ & -.004 & $.135^{*}$ & $\begin{array}{c}.324 * * \\
*\end{array}$ & $\begin{array}{c}.219 * * \\
*\end{array}$ & .093 & $\begin{array}{c}.608^{* *} \\
*\end{array}$ & - \\
\hline 10. BMI & .050 & -.025 & -.002 & .016 & $.152 * *$ & $.130 *$ & -.016 & $\begin{array}{c}.212^{* * *} \\
*\end{array}$ & $\begin{array}{c}.200 * * \\
*\end{array}$ \\
\hline
\end{tabular}

DIF = difficulty identifying feelings, DDF = difficulty describing feelings, EOT = externally oriented thinking, TAS-20 = total alexithymia scores, $\mathrm{NU}=$ negative urgency, $\mathrm{BDI}=\mathrm{Beck}$ depression inventory, BAI $=$ Beck anxiety inventory, EES = total emotional eating scale, TFEQ-EE = emotional eating subscale from the TFEQ, BMI = body mass index.

$*=p<.05, * *=p<.01, * * *=p \leq .001$

Serial Mediation Models

Depression. The models presented in Table 1 were also tested in Study Two. Model 1 revealed no significant direct effects between total TAS-20 scores and BMI, but a significant specific indirect effect was found via BDI scores $\left(a_{l} b_{1}\right), B=.0335, C I=.0019-.0660$. Models 
$.0795, C I=.0103-.1531$, model 3: $B=.0786, C I=.0097-.1518$, model 4: $B=.0214, C I=$

472

473

474

475

476

.0008-.0609. A similar pattern of results was found when emotional eating scores (EES) were entered (models 5-7). A significant specific indirect effect was found via BDI scores $\left(a_{1} b_{1}\right)$ : Model 5: $B=.0317, C I=.0011-.0649$; Model 6: $B=.0756, C I=.0093-.1481$, and Model 7: $B=.0734, C I=.0062-.1472)$. There were no significant direct or indirect pathways in model 8.

Anxiety. BAI scores were then entered as the measure of affect at $M_{1}$ for models 9-16. Model 9 found no significant direct pathway but a significant specific indirect pathway via BAI and NU, $\left(a_{1} d_{21} b_{2}\right), B=.0021, C I=.0001-.0066$. There was no significant direct or indirect effect for Model 10. There were two significant specific indirect pathways for Model 11 , via BAI and NU $\left(a_{1} d_{21} b_{2}\right), B=.0072, C I=.0008-.0189$, and via NU $\left(a_{2} b_{2}\right), B=.0341, C I$ $=.0019-.0787$. Contrast analysis revealed that the effect between these two indirect effects was significant and the indirect effect via NU was the strongest, $(B=.0269, C I=-.0693-$ $.0023)$. Model 12 revealed a significant indirect effect via NU $\left(a_{2} b_{2}\right), B=.0007, C I=.0004-$ .0446 and a second approached significance, via BAI, NU and TFEQ-EE $\left(a_{1} d_{21} d_{32} b_{3}\right), B=$ $.0001, C I=.0000-.0012$. When entering EES at $M_{3}$, a similar pattern of results to TFEQ-EE were found.

Our analysis revealed that when depression was included in the model, depression significantly mediated the effect of alexithymia on BMI. This remained significant when the DIF, DDF and EOT subscales of the TAS-20 were entered as the predictor variables. When including anxiety scores in the model, the indirect effect via negative urgency was the strongest mediating factor between alexithymia and BMI scores. 
We aimed to further understand the role of alexithymia as an explanatory mechanism in emotional eating, and in turn, BMI. Guided by previous literature, we put forward a novel theoretical model suggesting that alexithymia is indirectly associated with BMI through negative affect (anxiety and depression examined separately), impulsivity (negative urgency) and emotional eating (see Figure 1). Specifically, we proposed that alexithymia would be associated with greater levels of negative affect, leading individuals to act rashly in response (negative urgency) to avoid the associated sensations and to engage in emotional eating, leading to an increased BMI. Initially we tested our model in a student sample (Study One) before completing a self-replication to test the applicability of the model in a more representative general population sample (Study Two).

Within our student sample we identified several significant pathways between alexithymia and BMI. For models defining negative affect as depression, there was a direct effect between difficulty identifying feelings (DIF) and BMI, and an indirect effect through increased levels of depression, negative urgency and emotional eating. For models defining negative affect as anxiety, we found that difficulty describing feelings (DDF) indirectly predicted BMI through anxiety alone, but also through anxiety, negative urgency and emotional eating. In addition, indirect effects through negative urgency and emotional eating were found for total alexithymia DIF and DDF scores. In contrast, two main pathways were identified in our general population sample (Study Two). When negative affect was defined as depression, it mediated the relationship between total and subscale scores of alexithymia and BMI. In contrast, when negative affect was defined as anxiety, negative urgency significantly mediated the pathway between DDF, externally oriented thinking (EOT) and BMI.

First, it appears that the pathways between alexithymia and BMI seem to differ depending on whether negative affect is represented by anxiety or depression. This is not 
entirely surprising, as even though alexithymia is related to negative affect overall (Suslow \& Donges, 2017), its relationship with anxiety and depression is thought to differ (Hendryx, Haviland, \& Shaw, 1991; Marchesi, Brusamonti, \& Maggini, 2000). For example, Eizaguirre and colleagues (2004) found that depression was a significant predictor of alexithymia total and subscale scores in individuals with eating disorders, whereas anxiety was only a predictor of total alexithymia and DIF subscale scores when combined with depression. This indicates that depression may have stronger associations with alexithymia, which is supported by our findings and is consistent with the view that depression and alexithymia may be overlapping constructs (Marchesi et al., 2000; Honkalampi, Hintikka, Laukkanen, \& Viiamäki, 2001; Corcos et al., 2000; Parker, Bagby, \& Taylor, 1991). In support of this argument, Torres and colleagues (2015) previously highlighted how both constructs share multiple characteristics, including negative affect (Mattila et al., 2008), decreased ability to communicate affect to other people (Saarijarvi et al., 2001), problems with interpersonal communication (Mattila et al., 2008), and lack of emotional clarity (Rude \& McCarthy, 2003). Our findings also reinforce the view that depression and anxiety should always be considered as distinct emotional states within eating behaviour research. For example, Goossens and colleagues (2009) argued that eating in response to anxiety reduces hyper arousal, whereas eating in response to depression increases positive mood. Studies have also suggested that emotional or binge eating may serve to reduce anxiety but may increase depression (Haedt-Matt \& Keel, 2011; Rosenbaum \& White, 2015), and Finch and Tomiyama (2015) found that emotional eating acted as a buffer to daily life stresses in women, but only when they did not have elevated depressive symptoms.

Second, we found that different facets of alexithymia produced different pathways. The DIF subscale of the TAS-20 directly and indirectly predicted BMI in the majority of models. Indeed, the only time that it did not play a role was when anxiety was included in the 
545 models in the general population sample. Overall, these findings emphasise the 546 multidimensional nature of alexithymia (Taylor, Bagby, Luminet, 2000) and suggest that 547 difficulty identifying feelings may be a core driving feature of alexithymia relevant to 548 emotional eating, and in turn, BMI. In support of this, Pinaquy et al. (2003) previously found 549 that DIF was the strongest predictor of emotional eating, and Larsen and colleagues (2006) 550 also found that DIF significantly mediated the relationship between depression and emotional eating alongside impulsivity.

Third, and as summarised above, we also found different pathways between alexithymia and BMI in our student versus general population sample, a finding that does not seem to be attributable to demographic differences across groups. For example, even though age, as well as levels of cognitive restraint and uncontrolled eating differed across our two samples, we controlled for these variables in our analyses. Although, it is possible that other demographic differences may have existed between our samples that we failed to consider. It seems likely that years spent in education and socioeconomic status would have differed across our two samples; factors known to be associated with both alexithymia and the adoption of emotional regulation strategies (Lane, Sechrest, \& Riedel, 1998; Parker, Taylor, \& Bagby, 2001). Instead, the fact we found different pathways across our two samples highlights an important methodological consideration in the development of models. Behaviour research using human participants relies heavily on convenient student samples, but results may only be applicable to other student samples and may not generalise to a broader population. For this reason, completing a self-replication as we have done here, should be seen as a pivotal step in similar research in the future. Therefore, although we

567 found contrasting results, it nevertheless highlights how important it is that initial models are interpreted with caution until they undergo rigorous testing in broader samples. 

eating is one of many contributing factors to increased BMI and obesity, and if emotional eating is a form of regulating emotions, then understanding the role of alexithymia and emotional dysregulation more broadly, will help develop strategies designed to assist weight

573 loss and management. For example, Finch and Tomiyama (2015) previously found that 574 comfort eating buffered the association between adverse life events and perceived stress, but only in individuals without elevated levels of depressive symptoms. This suggests that comfort eating in response to naturally occurring stressors may serve as a protective mechanism in some individuals. Therefore, whilst it is important to reduce emotional eating due to its associated negative impact on health, a new strategy is needed to help such individuals manage their response to stress and negative emotions. Weight management is likely to differ if emotional eating is withdrawn as a coping strategy, as this would also remove the use of food to self-soothe. Therefore, addressing an individual's ability to identify, describe and regulate emotions should be considered when designing interventions to manage weight. female. This may influence results as males are reported to exhibit greater levels of alexithymia than their female counterparts (Mattila, Salminen, Nummi \& Joukamaa, 2006;

587 Honkalampi, Hintikka, Tanskanen, Lehtonen, \& Viianamäki, 2000), as well as lower levels of depression and emotional eating (e.g. Larsen et al., 2006). Therefore, it is possible that the pathways between alexithymia and BMI may differ across genders and this should be explored in future research. Second, even though we controlled for age in our analyses, we did not control for the possible influence of age related factors, such as the use of more adaptive and less impulsive coping strategies over the course of one's lifespan (Diehl, Coyle, 
However, whilst some studies suggest that self-report methods can lead to an underestimation and overestimation of weight and height, respectively (Spencer, Appleby, Davey and Key, 2001), self-reported body weight has been found to be an excellent approximation of actual body weight across a broad range of populations (Jeffrey, 1996; Kuczmarkski, Kuczmarkski, and Najjar, 2001). Fourth, even though our choice of mediating factors was guided by previous literature, it is possible that other factors may also play a role. For instance, anxiety sensitivity, the belief that sensations associated with anxiety have negative consequences, has been associated with alexithymia (Devine, Stewart, \& Watt, 1999; Wood, O'Hagan, Williams, McCabe, \& Chadwick, 2014). It may be that individuals who are uncomfortable with the associated sensations of anxiety may be more inclined to try to remove them as quickly as possible through maladaptive coping strategies, such as emotional eating (Reaves, Christiansen, Boyland, Halford, Llewellyn, \& Hardman, 2016). Interoceptive awareness, defined as the ability to accurately detect and interpret bodily sensations and associated with alexithymia, may also be an important mediating factor (Herbert, Herbert, \& Pollatos, 2011). Individuals who are have low interoceptive awareness may be vulnerable to engaging in emotional eating due to misinterpretation of bodily sensations of arousal (e,g. butterflies in the stomach associated with anxiety interpreted as pangs of hunger; Young, Williams, Pink, Freegard, Owens, \& Benton, 2017). Future research should explore whether these variables further mediate the relationship alexithymia and BMI. Finally, and consistent with previous research (Pinaquy et al. 2003; Pike 2013; Larsen et al., 2006), the internal consistency of the EOT subscale of the TAS-20 was relatively low across both of our samples. Consequently, conclusions drawn around this subscale should be interpreted with caution.

To conclude, our research offers novel insight into the relationship between alexithymia and BMI and highlights important methodological considerations for future research. For the first time, we incorporated multiple mediating factors (negative affect, negative urgency and 
619 emotional eating) in one inclusive theoretical model, and outcomes were tested for robustness 620 through self-replication in a more representative general population sample. Overall, our 621 results provide some support for the hypothesised model. Exploratory analysis in a student 622 sample revealed that difficulty identifying feelings predicted BMI both directly and indirectly 623 via negative urgency and emotional eating. In contrast, results from our more representative 624 general population sample revealed that alexithymia predicted BMI indirectly via depression 625 or impulsiveness. Whilst the precise nature in which alexithymia drives emotional eating 626 remains unclear, these findings represent the first steps in developing one inclusive model 627 and add strength to the proposal that alexithymia is an important factor in understanding 628 emotional eating and BMI. 
631

632

633

634

635

636

637

638

639

640

641

642

643

644

645

646

647

648

649

650

651

652

Arnow, B., Kenardy, J., \& Agras, W. S. (1995). The Emotional Eating Scale: The development of a measure to assess coping with negative affect by eating. Int $J$ Eat Disord, 18(1), 79-90. doi:10.1002/1098-108X(199507)18:1<79::AIDEAT2260180109>3.0.CO;2-V

Bagby, R. M., Parker, J. D., \& Taylor, G. J. (1994). The twenty-item Toronto Alexithymia Scale-I. Item selection and cross-validation of the factor structure. J Psychosom Res, 38(1), 23-32. doi:10.1016/0022-3999(94)90005-1

Bagby, R. M., Taylor, G. J., \& Parker, J. D. (1994). The twenty-item Toronto Alexithymia Scale-II. Convergent, discriminant, and concurrent validity. J Psychosom Res, 38(1), 33-40. doi:10.1016/0022-3999(94)90006-X

Beck, A. T., Epstein, N., Brown, G., \& Steer, R. A. (1988a). An inventory for measuring clinical anxiety: psychometric properties. J Consul Clin Psychol, 56(6), 893. doi:10.1037/0022-006X.56.6.893

Beck, A. T., \& Steer, R. A. (1990). Manual for the Beck anxiety inventory. San Antonio, USA: The Psychological Corporation.

Beck, A. T., Steer, R. A., \& Brown, G. K. (1996). Beck depression inventory-II. San Antonio, USA: The Psychological Corporation

Beck, A. T., Steer, R. A., \& Carbin, M. G. (1988). Psychometric properties of the Beck Depression Inventory: Twenty-five years of evaluation. Clin Psychol Rev, 8(1), 77-100. doi:10.1016/0272-7358(88)90050-5

Beck, A. T., Ward, C. H., Mendelson, M., Mock, J., \& Erbaugh, J. (1961). An inventory for measuring. Arch Gen Psych, 4, 561-571. doi:10.1001/archpsyc.1961.01710120031004 
Berardis, D. D., Campanella, D., Nicola, S., Gianna, S., Alessandro, C., Chiara, C., ... \& Ferro, F. M. (2008). The impact of alexithymia on anxiety disorders: a review of the literature. Curr Psych Rev, 4(2), 80-86. doi:10.2174/157340008784529287

Corcos, M., Guilbaud, O., Speranza, M., Paterniti, S., Loas, G., Stephan, P., \& Jeammet, P. (2000). Alexithymia and depression in eating disorders. Psychiatry Res, 93(3), 263-266. doi:10.1016/S0165-1781(00)00109-8

Cyders, M. A., Smith, G. T., Spillane, N. S., Fischer, S., Annus, A. M., \& Peterson, C. (2007). Integration of impulsivity and positive mood to predict risky behavior: Development and validation of a measure of positive urgency. Psychol Assess, 19(1), 107. doi:10.1037/1040-3590.19.1.107

De Lauzon, B., Romon, M., Deschamps, V., Lafay, L., Borys, J. M., Karlsson, J., ... \& Charles, M. A. (2004). The Three-Factor Eating Questionnaire-R18 is able to distinguish among different eating patterns in a general population. J Nutr, 134(9), 2372-2380.

Devine, H., Stewart, S. H., \& Watt, M. C. (1999). Relations between anxiety sensitivity and dimensions of alexithymia in a young adult sample. J Psychosom Res, 47(2), 145-158. doi:10.1016/S0022-3999(99)00033-1

Diehl, M., Coyle, N., \& Labouvie-Vief, G. (1996). Age and sex differences in strategies of coping and defense across the life span. Psychol Aging, 11(1), 127.

Eizaguirre, A. E., de Cabezon, A. O. S., de Alda, I. O., Olariaga, L. J., \& Juaniz, M. (2004). Alexithymia and its relationships with anxiety and depression in eating disorders. Pers Individ Diff, 36(2), 321-331. doi:10.1016/S0191-8869(03)00099-0 
675 Fernandes, J., Ferreira-Santos, F., Miller, K., \& Torres, S. (2018). Emotional processing in

676

677

678

679

680

681

682

683

684

685

686

687

688

689

690

691

692

693

694

695

696 obesity: a systematic review and exploratory meta-analysis. Obes Rev 19(1), 111-120. doi: 10.1111/obr.12607

Finch, L. E., \& Tomiyama, A. J. (2015). Comfort eating, psychological stress, and depressive symptoms in young adult women. Appetite, 95, 239-244. doi:10.1016/j.appet.2015.07.017

Geliebter, A., \& Aversa, A. (2003). Emotional eating in overweight, normal weight, and underweight individuals. Eat Beh, 3(4), 341-347. doi:10.1016/S1471-0153(02)00100-9

Gunderson, J.G., \& Zanarini, M. C. (1989): Pathogenesis in borderline personality. In A. Tasman, R. E. Hales, \& A. J. Frances (Eds.), Review of Psychiatry, Vol. 8 (pp. 25-48). Washington, USA: American Psychiatric Press.

Haedt-Matt, A. A., Keel, P. K., Racine, S. E., Burt, S. A., Hu, J. Y., Boker, S., ... \& Klump, K. L. (2014). Do emotional eating urges regulate affect? Concurrent and prospective associations and implications for risk models of binge eating. Int J Eat Disord, 47(8), 874-877. doi:10.1002/eat.22247

Hayes, A. F. (2012). Introduction to mediation, moderation, and conditional process analysis: A regression-based approach. New York, USA: Guilford Publications.

Heatherton, T. F., \& Baumeister, R. F. (1991). Binge eating as escape from selfawareness. Psychol Bull, 110(1), 86.

Hendryx, M. S., Haviland, M. G., \& Shaw, D. G. (1991). Dimensions of alexithymia and their relationships to anxiety and depression. J Pers Assess, 56(2), 227-237. doi:10.1207/s15327752jpa5602_4 
Herbert, B. M., Herbert, C., \& Pollatos, O. (2011). On the relationship between interoceptive awareness and alexithymia: is interoceptive awareness related to emotional awareness? J Pers, 79(5), 1149-1175. doi:10.1111/j.1467-494.2011.00717.x

Holliday, J., Uher, R., Landau, S., Collier, D., \& Treasure, J. (2006). Personality pathology among individuals with a lifetime history of anorexia nervosa. J Pers Disord, 20(4), 417-430. doi:10.152/pedi.2006.20.4.417

Honkalampi, K., Hintikka, J., Tanskanen, A., Lehtonen, J., \& Viinamäki, H. (2000). Depression is strongly associated with alexithymia in the general population. $J$ Psychosom Res, 48(1), 99-104. doi:10.1016/S0022-3999(99)00083-5

Honkalampi, K., Hintikka, J., Laukkanen, E., \& Viinamäki, J. L. H. (2001). Alexithymia and depression: a prospective study of patients with major depressive disorder. Psychosomatics, 42(3), 229-234. doi:10.1176/appi.psy.42.3.229

Jasinska, A. J., Yasuda, M., Burant, C. F., Gregor, N., Khatri, S., Sweet, M., \& Falk, E. B. (2012). Impulsivity and inhibitory control deficits are associated with unhealthy eating in young adults. Appetite, 59(3), 738-747. doi:10.1016/j.appet.2013.08.001

Jeffery, R. (1996). Bias in reported body weight as a function of education, occupation, health and weight concern. (1996). Add Beh, 21(2), 217-222. doi: 10.1016/03064603(95)00050-X

Kang, J. I., Namkoong, K., Yoo, S. W., Jhung, K., \& Kim, S. J. (2012). Abnormalities of emotional awareness and perception in patients with obsessive-compulsive disorder. $J$ Affect Disord, 141(2), 286-293. doi:10.1016/j.jad.2012.04.001 
Kaplan, H. I., \& Kaplan, H. S. (1957). The psychosomatic concept of obesity. J Nerv Ment Dis, 125, 181-201.

Karlsson, J., Persson, L. O., Sjöström, L., \& Sullivan, M. (2000). Psychometric properties and factor structure of the Three-Factor Eating Questionnaire (TFEQ) in obese men and women. Results from the Swedish Obese Subjects (SOS) study. Int J Obes, 24(12), 1715. doi:10.1038/sj.ijo.0801442

Koenders, P. G., \& van Strien, T. (2011). Emotional eating, rather than lifestyle behavior, drives weight gain in a prospective study in 1562 employees. J Occup Environ Med, 53(11), 1287-1293. doi:10.1097/JOM.0b013e31823078a2

Konttinen, H., Männistö, S., Sarlio-Lähteenkorva, S., Silventoinen, K., \& Haukkala, A. (2010). Emotional eating, depressive symptoms and self-reported food consumption. A population-based study. Appetite, 54(3), 473-479. doi:10.1016/J.APPET.2010.01.014

Kuczmarski, M. F., Kuczmarski, R. J., \& Najjar, M. (2001). Effects of age on validity of selfreported height, weight, and body mass index: findings from the Third National Health and Nutrition Examination Survey, 1988-1994. J Am Diet AssOC, 101(1), 28-34. doi: $10.1016 / \mathrm{S} 0002-8223(01) 00008-6$

Lane, R. D., Sechrest, L., \& Riedel, R. (1998). Sociodemographic correlates of alexithymia. Compr Psychiatry, 39(6), 377-385.

Lane, R. D., Lee, S., Reidel, R., Weldon, V., Kaszniak, A., \& Schwartz, G. E. (1996). Impaired verbal and nonverbal emotion recognition in alexithymia. Psychosom Med, 58(3), 203-210. 
739

740

741

742

743

744

745

746

747

748

749

750

751

752

753

754

755

756

757

758

Larsen, J. K., van Strien, T., Eisinga, R., \& Engels, R. C. (2006). Gender differences in the association between alexithymia and emotional eating in obese individuals. $J$ Psychosom Res, 60(3), 237-243. doi:10.1016/J.JPSYCHORES.2.005.07.006

Lehman, A. K., \& Rodin, J. (1989). Styles of self-nurturance and disordered eating. J Consult Clin Psychol, 57(1), 117. doi:10.1037/0022-006X.57.1.117

Linehan, M. M. (1993). Cognitive-Behavioral Treatment of Borderline Personality Disorder. New York, USA: The Guilford Press.

Linehan, M. M. (1995). Understanding Borderline Personality Disorder. New York, USA: The Guilford Press.

Lluch, A., Herbeth, B., Méjean, L., \& Siest, G. (2000). Dietary intakes, eating style and overweight in the Stanislas Family Study. Int J Obes, 24(11), 1493-1499. doi:10.1038/sj.ijo.0801425

Macht, M., \& Simons, G. (2000). Emotions and eating in everyday life. Appetite, 35(1), 6571. doi:10.1006/appe.2000.0325

Marchesi, C., Brusamonti, E., \& Maggini, C. (2000). Are alexithymia, depression, and anxiety distinct constructs in affective disorders? J Psychosom Res, 49(1), 43-49. doi:10.1016/S0022-3999(00)00084-2

Mattila, A. K., Poutanen, O., Koivisto A-M., Salokangas, R. K. R., \& Joukamaa, M. (2008). The performance of diagnostic measures of depression in alexithymic and nonalexithymic subjects. Gen Hos Psych, 30 (1), 77-79 
Mattila, A. K., Salminen, J. K., Nummi, T., \& Joukamaa, M. (2006). Age is strongly associated with alexithymia in the general population. J Psychosom Res, 61(5), 629635. doi:10.1016/J.PSYCHORES.2.006.04.013

Moon, A., \& Berenbaum, H. (2009). Emotional awareness and emotional eating. Cogn Emot 23(3), 417-429. doi:10.1080/02699930801961798

Noli, G., Cornicelli, M., Marinari, G. M., Carlini, F., Scopinaro, N., \& Adami, G. F. (2010). Alexithymia and eating behaviour in severely obese patients. J Hum Nutr Diet, 23(6), 616-619. doi:10.1111/j.1365-277X.2010.01079.x

Ouwens, M. A., van Strien, T., \& van Leeuwe, J. F. (2009). Possible pathways between depression, emotional and external eating. A structural equation model. Appetite, 53(2), 245-248. doi:10.1016/J.APPET.2009.06.001

Parker, J. D., Bagby, R. M., \& Taylor, G. J. (1991). Alexithymia and depression: distinct or overlapping constructs? Compr Psychiatry, 32(5), 387-394. doi:10.1016/0010$440 X(91) 90015-5$

Parker, J. D., Taylor, G. J., \& Bagby, R. M. (2001). The relationship between emotional intelligence and alexithymia. Pers Individ Diff, 30(1), 107-115. doi:10.1016/S01918869(00)000014-3

Parker, J. D., Taylor, G. J., \& Bagby, R. M. (2003). The 20-Item Toronto Alexithymia Scale: III. Reliability and factorial validity in a community population. $J$ Psychosom Res, 55(3), 269-275. doi:10.1016/S0022-3999(02)00578-0 
Pike, C. (2013). The Association between Alexithymia, Impulsivity and Negative Affect in Emotional and External Eating. (Master's thesis). Retrieved from https://ir.canterbury.ac.nz/bitstream/handle/10092/8986/thesis_fulltext.pdf?sequence=1

Pinaquy, S., Chabrol, H., Simon, C., Louvet, J. P., \& Barbe, P. (2003). Emotional Eating, Alexithymia, and Binge-Eating Disorder in Obese Women. Obes Res, 11(2), 195-201. doi:10.1038/oby.2003.31

Polivy, J., \& Herman, C. P. (1999). Distress and eating: why do dieters overeat? Int J Eat Disord, 26(2), 153-164. doi:10.1002/(SICI)1098-108X(199909026:2<153::AIDEAT4>3.0.CO;2-R

Price, M., Higgs, S., \& Lee, M. (2015). Self-reported eating traits: underlying components of food responsivity and dietary restriction are positively related to BMI. Appetite, 95, 203-210. doi:10.1016/J.APPET.2015.07.006

Racine, S. E., Keel, P. K., Burt, S. A., Sisk, C. L., Neale, M., Boker, S., \& Klump, K. L. (2013). Exploring the relationship between negative urgency and dysregulated eating: Etiologic associations and the role of negative affect. J Abnorm Psychol, 122(2), 433. doi:10.1037/a0031250

Reaves, D. L., Christiansen, P., Boyland, E., Halford, J. C. G., Llewellyn, C. H., \& Hardman, C. A. (2016). Cross-sectional associations between personality, eating to cope, and consumption. Appetite, (107), 689-690. doi:10.1016/j.appet.2016.08.076

Rosenbaum, D. L., \& White, K. S. (2015). The relation of anxiety, depression, and stress to binge eating behavior. J Health Psychol, 20(6), 887-898.

doi:10.1177/1359105315580212 
801

802

803

804

805

806

807

808

809

810

811

812

813

814

815

816

817

818

819

820

Rude, S., \& McCarthy, C. (2003). Emotional functioning in depressed and depressionvulnerable college students. Cog Emo, 17(5), 799-806.

Saarijärvi, S., Salminen, J. K., \& Toikka, T. B. (2001). Alexithymia and depression: a 1year follow-up study in outpatients with major depression. J Psychosom Res, 51(6), 729-733.

Salminen, J. J. K., Saarijärvi, S., Äärelä, E., Toikka, T., \& Kauhanen, J. (1999). Prevalence of alexithymia and its association with sociodemographic variables in the general population of finland. J Psychosom Res, 46(1), 75-82. doi:10.1016/S00223999(98)00053-1

Shishido, H., Gaher, R. M., \& Simons, J. S. (2013). I don't know how I feel, therefore I act: alexithymia, urgency, and alcohol problems. Addict Behav, 38(4), 2014-2017. doi:10.1016/J.ADDBEH.2012.12.014

Sifneos, P. E. (1973). The prevalence of 'alexithymic' characteristics in psychosomatic patients. Psychother Psychosom, 22(2-6), 255-262. doi:10.1159/000286529

Spence, S., \& Courbasson, C. (2012). The role of emotional dysregulation in concurrent eating disorders and substance use disorders. Eat Behav, 13(4), 382-385. doi:10.1016/J.EATBEH.2012.05.006

Spencer, E. A., Appleby, P. N., Davey, G. K., \& Key, T. J. (2002). Validity of self-reported height and weight in 4808 EPIC-Oxford participants. Pub Health Nut, 5(4), 561-565. doi: 10.1079/PHN2001322 
Stunkard, A. J., \& Messick, S. (1985). The three-factor eating questionnaire to measure dietary restraint, disinhibition and hunger. J Psychosom Res, 29(1), 71-83. doi:10.1016/0022-3999(85)90010-8

Sung, J., Lee, K., \& Song, Y. M. (2009). Relationship of eating behavior to long-term weight change and body mass index: The Healthy Twin study. Eat Weight Disord-ST, 14(2-3), e98-e105. doi:10.1007/BF03327806

Suslow, T., \& Donges, U. S. (2017). Alexithymia components are differentially related to explicit negative affect but not associated with explicit positive affect or implicit affectivity. Front Psychol, 8, 1758. doi:10.3389/fpsyg.2017.01758

Taylor, G. J., Bagby, R. M, \& Luminet, O. (2000). Assessment of alexithymia: Self-report and observer-rated measures. In R. Bar-on \& J. D. A. Parker (Eds.), The Handbook of Emotional Intelligence (pp. 301-319). San Francisco, USA: Jossey Bass.

Taylor, G. J., Bagby, R. M., \& Parker, J. D. (1997). Disorders of affect regulation: Alexithymia in medical and psychiatric illness. New York, USA: Cambridge University Press.

Taylor GJ, Bagby RM, Parker JDA (2006). The 20-Item Toronto Alexithymia Scale Manual. http://www.gtaylorpsychiatry.org/tas.htm

Torres, S., Guerra, M. P., Lencastre, L., Miller, K., Vieira, F. M., Roma-Torres, A., ... Costa, P. (2015). Alexithymia in anorexia nervosa: The mediating role of depression. Psych Res, 225(1), 99-107. doi:10.1016/j.psychres.2014.10.023 
van Strien, T., Frijters, J. E., Bergers, G. P., \& Defares, P. B. (1986). The Dutch Eating Behavior Questionnaire (DEBQ) for assessment of restrained, emotional, and external eating behavior. Int J Eat Disord, 5(2), 295-315. doi:10.1002/1098108X(198602)5:2<295::AID-EAT2260050209>3.0.CO;2-T

van Strien, T., Herman, C.P. and Verheijden, M.W., 2012. Eating style, overeating and weight gain. A prospective 2-year follow-up study in a representative Dutch sample. Appetite, 59(3), pp.782-789. doi:10.1016/J.APPET.2012.08.009

van Strien, T., Konttinen, H., Homberg, J. R., Engels, R. C., \& Winkens, L. H. (2016). Emotional eating as a mediator between depression and weight gain. Appetite, 100, 216-224. doi:10.1016/J.APPET.2016.02.034

van Strien, T., \& Ouwens, M. A. (2007). Effects of distress, alexithymia and impulsivity on eating. Eat Behav, 8(2), 251-257. doi:10.1016/J.EATBEH.2006.06.004

Whiteside, S. P., \& Lynam, D. R. (2001). The five factor model and impulsivity: Using a structural model of personality to understand impulsivity. Pers Individ Diff, 30(4), 669689. doi:10.1016/S0191-8869(00)00064-7

Wood, R. L., O’Hagan, G., Williams, C., McCabe, M., \& Chadwick, N. (2014). Anxiety Sensitivity and Alexithymia as Mediators of Postconcussion Syndrome Following Mild Traumatic Brain Injury. J Head Trauma Rehabil, 29(1), E9-E17. doi:10.1097/HTR.0b013e31827eabba

Wood, R. L., Williams, C., \& Lewis, R. (2010). Role of alexithymia in suicide ideation after traumatic brain injury. J Int Neuropsychol Soc, 16(6), 1108-1114. doi:10.1017/S1355617710001013 
864 Young, H. A., Williams, C., Pink, A. E., Freegard, G., Owens, A., \& Benton, D. (2017).

865 Getting to the heart of the matter: Does aberrant interoceptive processing contribute

$866 \quad$ towards emotional eating? PloS one, 12(10), e0186312.

867 doi:10.1371/journal.pone.0186312

868 Źak-Golab, A., Tomalski, R., Bąk-Sosnowska, M., Holecki, M., Kocełak, P., Olszanecka-

869 Glinianowicz, M., Chudek, J., \& Zahorska-Markiewicz, B. (2103). Alexithymia,

870 depression, anxiety and binge eating in obese women. Eur J Psychiat, 27(3), 149-159.

871 doi: $10.4321 / \mathrm{S} 0213-61632013000300001$

872 